A BROADBAND COMBINED (LINEAR AND LOOP) ANTENNA ABOVE A GROUND

PLANE

\author{
Karlo Queiroz da Costa ${ }^{(1)}$, Victor Dmitriev $^{(1)}$, and Anderson Oliveira Silva ${ }^{(1)}$ \\ ${ }^{(1)}$ Department of Electrical Engineering and Computation, Federal University of Para \\ Av. Augusto Corrêa n ${ }^{\circ}$ 01, CEP 66075-900, Belém-PA, Brazil \\ Emails: karlocosta@yahoo.com.br, victor@ufpa.br, anderson.osilva@gmail.com
}

\title{
INTRODUCTION
}

Antennas that possess broadband characteristics, high radiation efficiency and low dimensions are important in practical applications, for example in mobile communication systems. Wide bandwidth is fundamental in antennas for transmitting broadband signals, in particular, video signals and signals with high transmition rate. In antenna theory, it is well known that small antennas possess narrow bandwidth and small radiation resistance, i.e. small radiation efficiency [1].

General fundamental limits of the antenna performances with respect to the parameters mentioned above were firstly established by Wheeler [2] and Chu [3]. These authors obtained the identical results about the effect of the dimension reduction on the bandwidth. One of their conclusions is that the minimum radiation factor that any antenna can have is only possible when this antenna radiates the fundamental modes $\mathrm{TM}_{10}$ e $\mathrm{TE}_{10}$ with equal energy. The fields of these modes are the same that those of the infinitesimal electric and magnetic dipoles, respectively. One can say that a smaller radiation factor leads to a larger bandwidth [3]. Collin [4] in 1964 confirmed the Chu results using field theory to compute the radiating energies. Fante [5] in 1969, generalized the ideas of Collin to an antenna that radiates any spherical mode. In [6], McLean made a verification of these fundamental limits using infinitesimal dipoles, and he suggested a simpler method to obtain the limits. Recent results that confirm these theories can be found in [7]-[9].

Four types of combinations of electric and magnetic dipoles in free space and with single feeding are presented in [10]. The authors showed theoretically that these antennas can possess broadband matching with the bandwidth about $86 \%$. The electric and magnetic dipoles should be combined in such a manner that their moments are orthogonal. The interaction between them (coupling) is stronger for such orientation of the dipoles.

In this work, we present three types of broadband combinations of linear and loop antennas with single feeding placed above a perfect ground plane. These antennas are analyzed by the Method of Moments (MoM) [11] and the antenna's dimensions are optimized to obtain a large matching bandwidth. We calculate the reflection coefficient, gain, and radiation diagrams of these antennas.

\section{DESCRIPTION OF THE ANTENNAS}

The geometries of the analyzed antennas are shown in Fig. 1. In all these configurations, the source is put in the middle of the electric linear antenna (dipole) and the loops are near this dipole with only electromagnetic coupling between them. In this figure, square loops are shown. To comparison, the configurations with circular loops are also analyzed. In these cases, the diameter of the circular loop is equal to the side of the corresponding square loop.

In all of these antennas, $L_{d}$ is the length of the electric dipole and $L_{e}$ is the side of a square loop (or diameter in the case of circular loop). In the first case shown in Fig. 1(a), we use one electric dipole and one loop. These two elements are centralized with respect to the origin of the axes $x$ and $y$, but they lie in different planes along the $z$-axis. The first element is in the plane $z=h_{d}$ and the second one in the plane $z=h_{e}$. Fig. 1(b) also shows a combination of one electric dipole and one loop for different position of the loop. The distance between the loop and the dipole is $d_{1}$. In Fig. 1(c), there is one electric dipole and two loops. These three elements are centralized with respect to the axis $x$ and $y$, but in different planes along the $z$-axis. The electric dipole is in the plane $z=h_{d}$ and the loops are in the planes $z=h_{e l}$ and $z=h_{e l}$. 


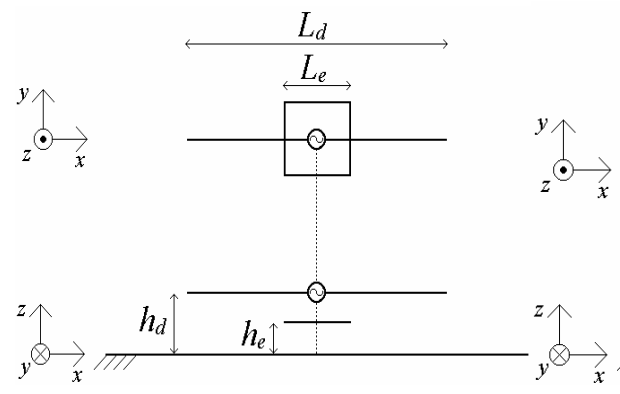

(a)

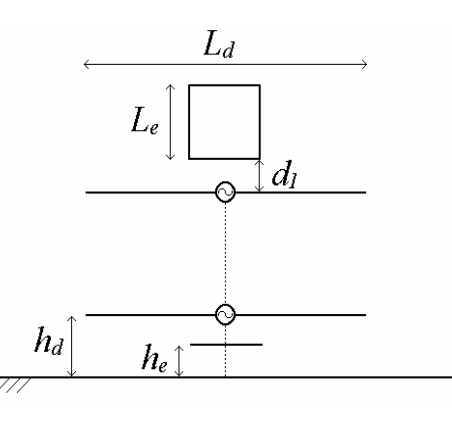

(b)

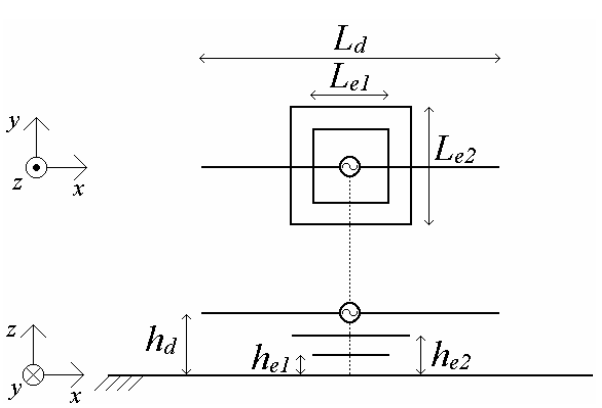

(c)

Fig. 1. Geometries of the three types of combination of linear and loop elements above a ground plane. (a) Case 1. (b) Case 2. (c) Case 3.

\section{NUMERICAL RESULTS}

The numerical analysis of these antennas is made by using MoM codes developed by Matlab 6. In this code, we use rectangular pulse for basis functions and the Dirac's functions for test functions. We use 15 segments of discretization for the horizontal electric dipole, 16 for the circular loops and 5 in each side of the square loops.

\section{Reflection Coefficient and Gain}

For the three antennas described above, we made many simulations with different values of the parameters $L_{e}, L_{e l}, L_{e 2}$, $h_{d}, h_{e l}, h_{e 2}$ and $d_{1}$, and the two types of loop (circular and square). In all of these simulations we fixed the height of the electric dipole in $h_{d}=0.3 L_{\mathrm{d}}$ and the radius of the transversal section of the conductors is $a=L_{d} / 200$. The antennas which had better results with respect to their bandwidths are presented in Table 1. The values of the parameters in this table are normalized to the length of the electric dipole $L_{d}$.

TABLE I DiMENSIONS OF THE ANTENNAS WITH LARGEST MATCHING BANDWIDTH

\begin{tabular}{|c|c|c|c|c|c|c|}
\hline & \multicolumn{2}{|c|}{ Case 1} & \multicolumn{2}{|c|}{ Case 2} & \multicolumn{2}{|c|}{ Case 3} \\
\hline & Circular & Square & Circular & Square & Circular & Square \\
\hline$L_{e}$ & 0.25 & 0.25 & 0.30 & 0.25 & - & - \\
\hline$L_{e l}$ & - & - & - & - & 0.25 & 0.20 \\
\hline$L_{e 2}$ & - & - & - & - & 0.25 & 0.25 \\
\hline$h_{e}$ & 0.35 & 0.35 & 0.27 & 0.27 & - & - \\
\hline$h_{e I}$ & - & - & - & - & 0.24 & 0.32 \\
\hline$h_{e 2}$ & - & - & - & - & 0.33 & 0.33 \\
\hline$d_{1}$ & - & - & 0.03 & 0.03 & - & - \\
\hline
\end{tabular}

The numerical results of the input matching $\left(\Gamma=\left|\left(Z_{i n}-Z_{0}\right) /\left(Z_{i n}+Z_{0}\right)\right|\right.$, where $Z_{0}$ is the transmission line impedance and $Z_{i n}$ is the input impedance) and gain are shown in Fig. 2. The gain is calculated for the direction normal to the ground plane $(+z)$. This gain is for the $x$-polarized wave. The gain of these antennas in direction $y$ is null. In this figure, the reflection coefficient of the isolated horizontal electric dipole is also shown for comparison. The isolated electric dipole has the length $L_{d}$ and the height $h_{d}=0.3 L_{d}$. The bandwidth of this dipole is $5 \%$ (with $Z_{0}=30 \mathrm{Ohms}$ ).

Table II shows the bandwidth of these optimized antennas $\left(B=200 \times\left(f_{s}-f_{i}\right) /\left(f_{s}+f_{i}\right)\right.$, where $f_{s}$ is the superior frequency and $f_{i}$ is the inferior frequency with respect the level of $-10 \mathrm{~dB}$ of the reflection coefficient). In this table, we present also the central normalized length $L_{d} / \lambda_{c}$, where $\lambda_{c}$ is the wavelength in the central frequency of the band $f_{c}=\left(f_{s}+f_{i}\right) / 2$, and the values of the characteristic line impedance $Z_{0}$ used for calculations. 


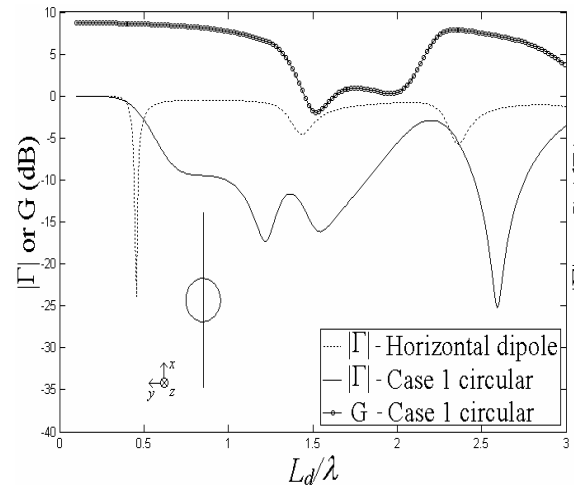

(a)

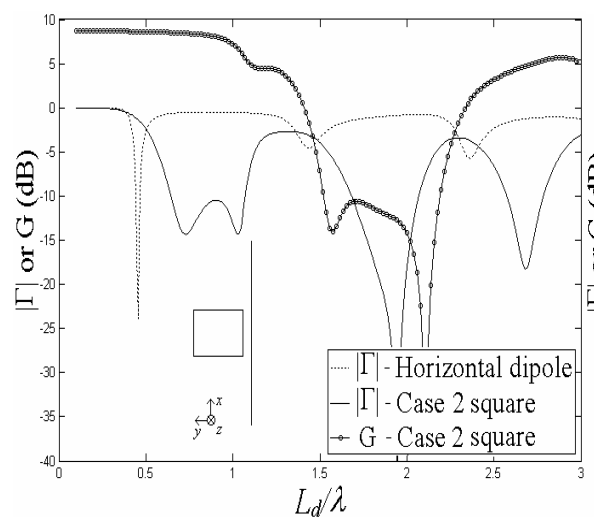

(d)

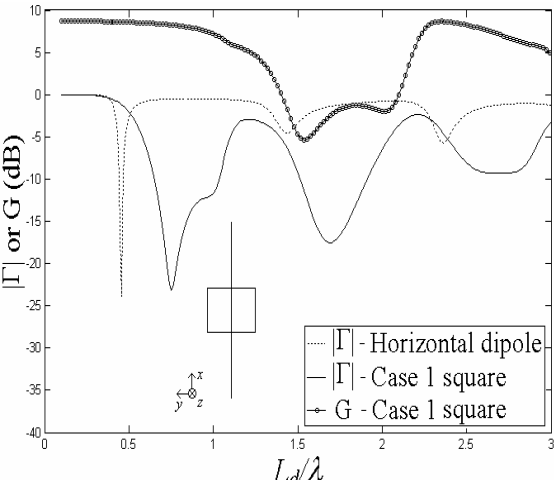

(b)

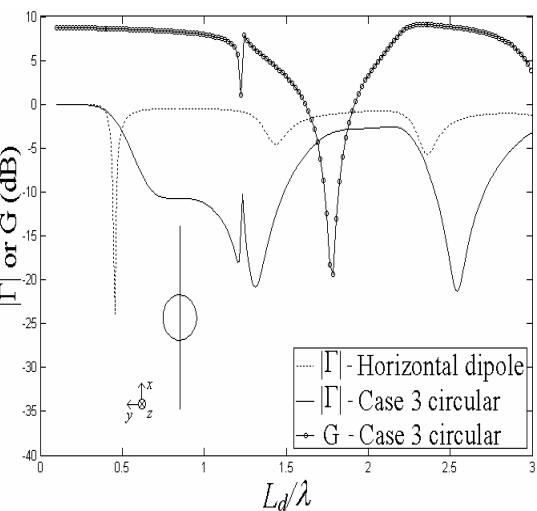

(e)

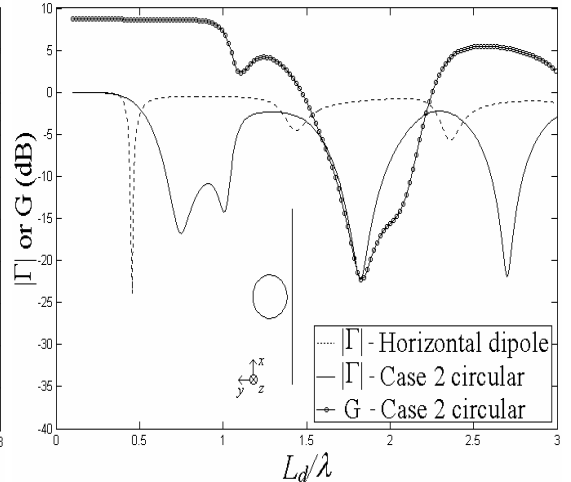

(c)

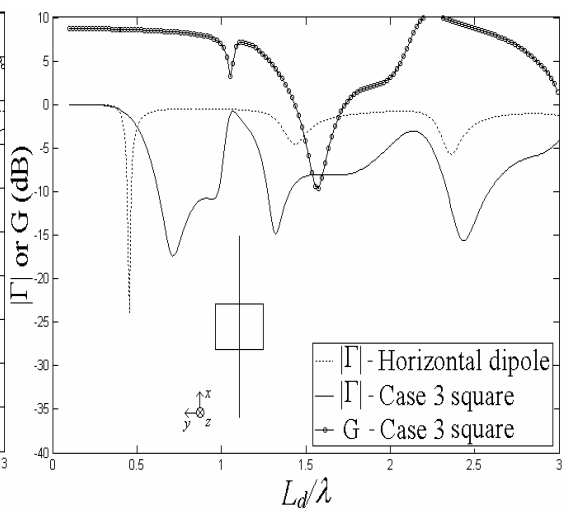

(f)

Fig. 2. Input impedance and gain of the optimized antennas. (a) Case 1 circular. (b) Case 1 square. (c) Case 2 circular. (d) Case 2 square. (e) Case 3 circular. (f) Case 3 square.

TABLE II BANDWIDTH $B$ OF THE OPTIMIZED ANTENNAS

\begin{tabular}{llccc}
\hline & \multicolumn{1}{c}{ Loop } & $B(\%)$ & $Z_{0}(\mathrm{Ohms})$ & $L_{d} / \lambda_{c}$ \\
\hline Case 1 & Circular & 60 & 350 & 1.41 \\
Case 1 & Square & 45 & 500 & 0.85 \\
Case 2 & Circular & 45 & 550 & 0.86 \\
Case 2 & Square & 49 & 450 & 0.87 \\
Case 3 & Circular & 73 & 320 & 1.01 \\
Case 3 & Square & 43 & 400 & 0.81 \\
\hline
\end{tabular}

\section{Radiation Diagrams}

The radiation diagrams of these optimized antennas (Table 1) are shown in Fig. 3, 4, 5, 6, 7 and 8. The frequencies $L_{d} / \lambda_{c}$ at which these diagrams were calculated, are given of in Table 2. In these figures, $\theta$ and $\phi$ are the usual spherical coordinates. These graphics correspond to the $x$ polarization. The component of the $y$ polarization is null.

The radiation diagram of the Case 1 (circular loop) on the plane $x z$ (Fig. 3(a)) has side lobes. This is because the value of the central wavelength $\left(\lambda_{c}=1.41\right)$ and the radiation diagram of a conventional electric dipole in this frequency has side lobes [1]. The radiation diagrams of the other antennas do not have side lobes. We also observe from Fig. 5(b) and Fig. 6(b) that the diagrams for these cases are asymmetric. This asymmetry exists in the plane $y z$. In the other cases, the diagrams possess symmetry in both the planes $x z$ and $y z$. These results are in agreement with the geometries of the antennas shown in Fig. 1. 


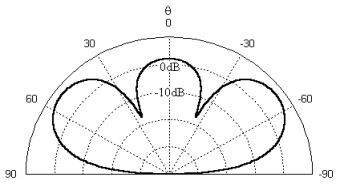

(a)

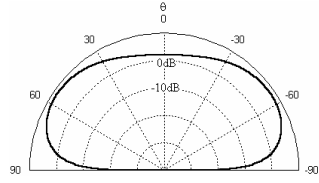

(b)

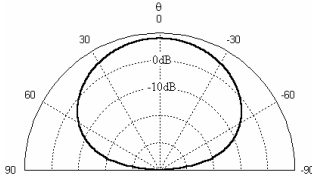

(a)

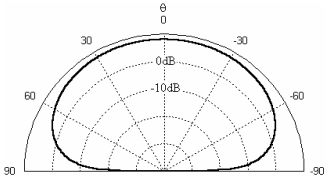

(b)

Fig. 3. Diagrams of the Case 1 circular. (a) Plane $x z$. (b) Plane $y z$. Fig. 4. Diagrams of the Case 1 square. (a) Plane $x z$. (b) Plane $y z$.

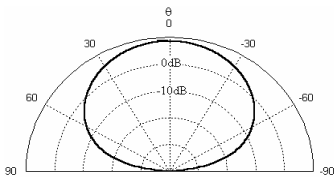

(a)

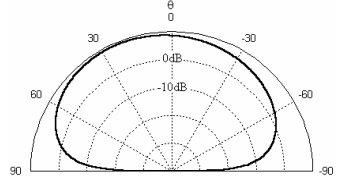

(b)

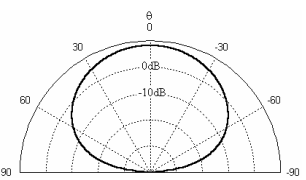

(a)

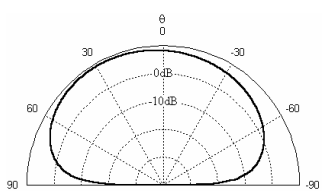

(b)

Fig. 5. Diagrams of the Case 2 circular. (a) Plane $x z$. (b) Plane $y z$. Fig. 6. Diagrams of the Case 2 square. (a) Plane $x z$. (b) Plane $y z$.

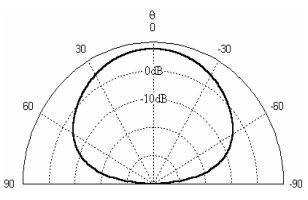

(a)

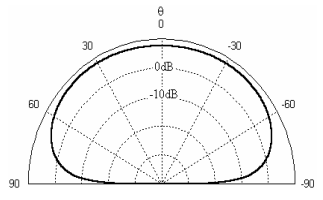

(b)

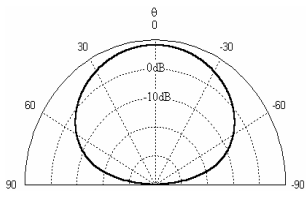

(a)

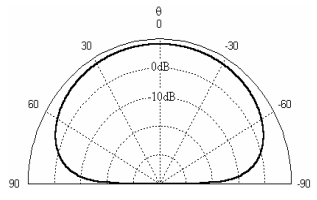

(b)

Fig. 7. Diagrams of the Case 3 circular. (a) Plane $x z$. (b) Plan $y z$. Fig. 8. Diagrams of the Case 3 square. (a) Plane $x z$. (b) Plane $y z$.

\section{CONCLUSIONS}

This work presents some preliminary results of optimization of combined antennas consisting of linear antenna and loops placed above an infinite ground plane. The obtained results show the input matching bandwidth up to $73 \%$ for the fixed height of $h_{d}=0.3 L_{d}$. We observed that the bandwidth for smaller height $h_{d}$ is diminished. In all the simulations, the obtained bandwidth is in the range $20 \%<B<40 \%$. The results show also that these types of antennas possess linear polarization in the direction normal to the ground plane and the values of the gain in this direction are usually larger than $5 \mathrm{~dB}$ in the range of matching $|\Gamma|<-10 \mathrm{~dB}$.

\section{REFERENCES}

[1] C. A. Balanis, Antenna Theory: Analysis and Design, 3nd ed., Ed. New York: John Wiley, 2005.

[2] H. A. Wheeler, "Fundamental limitations of small antenna", Proc. IRE, vol. 35, pp. 1479-1484, December 1947.

[3] L. J. Chu, "Physical limitations of omni-directional antennas", J. Appl. Phys., vol. 19, pp. 1163-1175, December 1948.

[4] R. E. Collin, S. Rothschild, "Evaluation of antenna Q", IEEE Trans. Ant. Propag., vol. AP-12, pp. 23-27, January 1964.

[5] R. L. Fante, “Quality factor of general ideal antennas”, IEEE Trans. Ant. Propag., vol. AP-17, pp. 151-155, March 1969.

[6] J. S. McLean, “A re-examination of the fundamental limits on the radiation Q of electrically small antennas", IEEE Trans. Ant. Propag., vol. 44, N5, pp. 672-675, May 1996.

[7] W. Geyi, P. Jarmuszewski, Y. Qi, “The foster reactance theorem for antennas and radiation Q", IEEE Trans. Ant. Propag., vol. 48, N3, pp. 401-408, March 2000.

[8] W. Geyi, "Physical limitations of antenna", IEEE Trans. Ant. Propag., vol. 51, N8, pp. 2116-2123, August 2003.

[9] W. Geyi, "A method for the evaluation of small antennas Q", IEEE Trans. Ant. Propag., vol. 51, N8, pp. 21242129, August 2003.

[10] Karlo Q. da Costa, and Victor A. Dmitriev, "Combination of electric and magnetic dipoles with single feeding for broadband applications", in press.

[11] R. F. Harrington, Field Computation by Moment Method, Ed. New York: Macmillan, 1968, pp. 62-81. 